\title{
ROBOTIC REVERBERATION MAPPING OF ARP 151
}

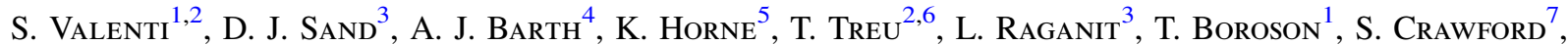 \\ A. Pancoast ${ }^{8,12}$, L. Pei ${ }^{4}$, E. Romero-Colmenero ${ }^{7,9}$, C. Villforth ${ }^{5,10}$, and H. Winkler ${ }^{11}$ \\ ${ }^{1}$ Las Cumbres Observatory Global Telescope Network, 6740 Cortona Drive, Suite 102, Goleta, CA 93117, USA \\ 2 Department of Physics, University of California, Santa Barbara, Broida Hall, Mail Code 9530, Santa Barbara, CA 93106-9530, USA \\ ${ }^{3}$ Texas Tech University, Physics Department, Box 41051, Lubbock, TX 79409-1051, USA \\ ${ }^{4}$ Department of Physics and Astronomy, University of California, 4129 Frederick Reines Hall, Irvine, CA, 92697-4575, USA \\ ${ }^{5}$ SUPA School of Physics \& Astronomy, University of St. Andrews, North Haugh KY16 9SS, UK \\ ${ }^{6}$ Department of Physics and Astronomy, University of California, Los Angeles, CA 90095-1547, USA \\ ${ }^{7}$ South African Astronomical Observatory, P.O. Box 9, Observatory 7935, Cape Town, South Africa \\ ${ }^{8}$ Harvard-Smithsonian Center for Astrophysics 60 Garden St Cambridge, MA 02138 USA \\ ${ }^{9}$ Southern African Large Telescope Foundation, P.O. Box 9, Observatory 7935, Cape Town, South Africa \\ ${ }^{10}$ Department of Physics, University of Bath, Claverton Down Road, Bath BA2 7AY, UK \\ ${ }^{11}$ Department of Physics, University of Johannesburg, P.O. Box 524, 2006 Auckland Park, South Africa \\ Received 2015 October 5; accepted 2015 October 25; published 2015 November 5
}

\begin{abstract}
We present the first results from the Las Cumbres Observatory Global Telescope (LCOGT) Network's Active Galactic Nuclei (AGNs) Key Project, a large program devoted to using the robotic resources of LCOGT to perform time domain studies of active galaxies. We monitored the Seyfert 1 galaxy Arp 151 (Mrk 40) for 200 days with robotic imagers and with the FLOYDS robotic spectrograph at Faulkes Telescope North. Arp 151 was highly variable during this campaign, with $V$-band light curve variations of $\sim 0.3$ mag and $\mathrm{H} \beta$ flux changing by a factor of $\sim 3$. We measure robust time lags between the $V$-band continuum and the $\mathrm{H} \alpha, \mathrm{H} \beta$, and $\mathrm{H} \gamma$ emission lines, with $\tau_{\text {cen }}=13.89_{-1.41}^{+1.39}, 7.52_{-1.06}^{+1.43}$, and $7.40_{-1.32}^{+1.50}$ days, respectively. The lag for the He II $\lambda 4686$ emission line is unresolved. We measure a velocity-resolved lag for the $\mathrm{H} \beta$ line, which is clearly asymmetric with higher lags on the blue wing of the line that decline to the red, possibly indicative of radial inflow, and is similar in morphology to past observations of the $\mathrm{H} \beta$ transfer function shape. Assuming a virialization factor of $f=5.5$, we estimate a black hole mass of $M_{\mathrm{BH}}=6.2_{-1.2}^{+1.4} \times 10^{6} M_{\odot}$, also consistent with past measurements for this object. These results represent the first step to demonstrate the powerful robotic capabilities of LCOGT for long-term AGN time domain campaigns that human intensive programs cannot easily accomplish. Arp 151 is now one of just a few AGNs where the virial product is known to remain constant against substantial changes in $\mathrm{H} \beta$ lag and luminosity.
\end{abstract}

Key words: galaxies: active - galaxies: individual (Arp 151) - galaxies: nuclei - galaxies: Seyfert techniques: spectroscopic

\section{INTRODUCTION}

Measuring supermassive black hole masses $\left(M_{\mathrm{BH}}\right)$ as a function of galaxy mass and cosmic time is one of the great challenges in observational astrophysics. A vital technique for measuring $M_{\mathrm{BH}}$ is reverberation mapping of Type 1, active galactic nuclei (AGNs; Blandford \& McKee 1982; Peterson 1993). Reverberation mapping traces the time lag between variable continuum emission from the accretion disk and the larger gas clouds that this emission photoionizes. This results in broad emission-line variability lagging behind the continuum emission. The lag time provides a distance scale due to the light travel time between the continuum source and the broad-line region (BLR), while the broad-line Doppler width provides a velocity. From these and from assumptions about the geometry and kinematics of the BLR, one can infer $M_{\mathrm{BH}}$. Reverberation mapping has been applied to dozens of nearby AGNs (e.g., Peterson et al. 2004; Bentz et al. 2009, 2010b; Denney et al. 2010; Grier et al. 2012; Du et al. 2014), and the resulting $R_{\mathrm{BLR}}-L$ relation (e.g., Bentz et al. 2013) is the basis for the "single-epoch" virial mass methods employed to track the growth history and redshift evolution of supermassive black holes (for a recent review, see Shen 2013).

\footnotetext{
${ }^{12}$ Einstein Fellow.
}

Reverberation mapping campaigns are labor intensive and expensive in terms of telescope time and human effort. They often require telescope time allocations over multiple periods with classically scheduled facilities.

A potentially transformative facility for reverberation mapping and time domain studies is the Las Cumbres Observatory Global Telescope network (LCOGT; Brown et al. 2013). LCOGT is currently composed of nine $1 \mathrm{~m}$ imaging telescopes at sites around the globe and two $2 \mathrm{~m}$ Faulkes telescopes, equipped with imagers and the FLOYDS robotic spectrographs. LCOGT is completely robotic, from the scheduling of the telescopes to the placement of targets onto spectrograph slits. Facilities such as LCOGT are ideally suited to conduct intensive reverberation mapping campaigns, as well as persistent multi-season efforts on luminous or higherredshift AGNs.

The AGN Key Project (PI: K. Horne) has been allocated substantial resources on the LCOGT network to conduct several AGN studies, including reverberation mapping of both local and high-redshift objects. Here, we report our first results -an intensive $\sim 200$ day long reverberation mapping campaign of the well-studied, local Seyfert 1 galaxy Arp 151 (also known as Mrk 40). Arp 151 was selected as part of an initial sample of low-redshift AGNs at LCOGT based on its strong variability in past campaigns (Bentz et al. 2008; Barth et al. 
2015). Our all-robotic observations show a clear velocityresolved reverberation lag.

These data allow us to investigate the time variability of the BLR over a time much longer than the lag and to infer the black hole mass in Arp 151. We verify that the virial product remains constant independent of an AGN luminosity change and demonstrate the promise of AGN studies with the LCOGT network.

\section{PHOTOMETRY}

We obtained high signal-to-noise ratio $V$-band imaging of Arp 151 from 2014 November 11 to 2015 July 4 with neardaily cadence. Observations were carried out with the $1 \mathrm{~m}$ telescope at McDonald Observatory and the $2 \mathrm{~m}$ Faulkes Telescope North (FTN) at Haleakala, Hawaii. The McDonald $1 \mathrm{~m}$ telescope was outfitted with a SBIG STX-16803 CCD with a $15^{\prime} .8 \times 15^{\prime} .8$ field of view, sampled with 0 ". 464 pixels. The FTN observations were taken with the spectral camera, with a $10.5 \times 10$. 5 field of view and a binned pixel scale of 0 !" 30 . Typical exposure times were $2 \times 120 \mathrm{~s}$ per visit.

Initial photometric data reduction, including overscan subtraction and flat fielding, was done via the LCOGT data pipeline (Brown et al. 2013). After this, the images were cleaned of cosmic rays using the L.A. Cosmic algorithm (van Dokkum 2001). In order to register the celestial coordinates across the set of images, we used the Astrometry.net software package (Lang et al. 2010).

Aperture photometry was applied using the automated IDL procedures described by Pei et al. (2014), using four comparison stars to construct a relative light curve. An aperture radius of $5^{\prime \prime}$ and sky annulus radii of $15^{\prime \prime}-30^{\prime \prime}$ were used throughout. Photometry measurements from images taken within $6 \mathrm{hr}$ of each other were combined to produce a weighted average magnitude for the final light curve. No attempt was made to remove host-galaxy light from the AGN photometry. We then used the AAVSO Photometric All-sky Survey (APASS; Henden et al. 2012) to flux calibrate the data. The final $V$-band light curve of 119 data points exhibits a sharp flux peak with an amplitude of $\sim 0.3 \mathrm{mag}$, ideal for measurement of broad-line reverberation (Figure 1).

\section{SPECTROSCOPIC OBSERVATIONS AND REDUCTION}

FLOYDS robotic spectroscopic observations of Arp 151 were carried out between 2014 December 6 and 2015 June 5 . All observations were taken at the parallactic angle with a 1 ." $6 \times 30^{\prime \prime}$ slit and an exposure time of $3600 \mathrm{~s}$. In all, 55 spectra of Arp 151 contribute to our emission-line light curve analysis.

The FLOYDS spectrographs are a pair of nearly identical instruments at the Faulkes Telescopes North and South (Arp 151 spectra, however, were all observed with FTN). They operate totally robotically, drastically lowering the human effort necessary to conduct long and intensive reverberation mapping campaigns. Targets are automatically placed in the slit using an acquisition camera and a fast cross-correlation algorithm to recognize the field and place the science object in the slit. FLOYDS uses a low dispersion grating (235 lines $\mathrm{mm}^{-1}$ ) and a cross-dispersed prism to work in first and second order simultaneously, giving a single-shot wavelength coverage of $\sim 3200-10,000$. First-order wavelength coverage extends over $\sim 4800-10000 \AA$ with a pixel scale of

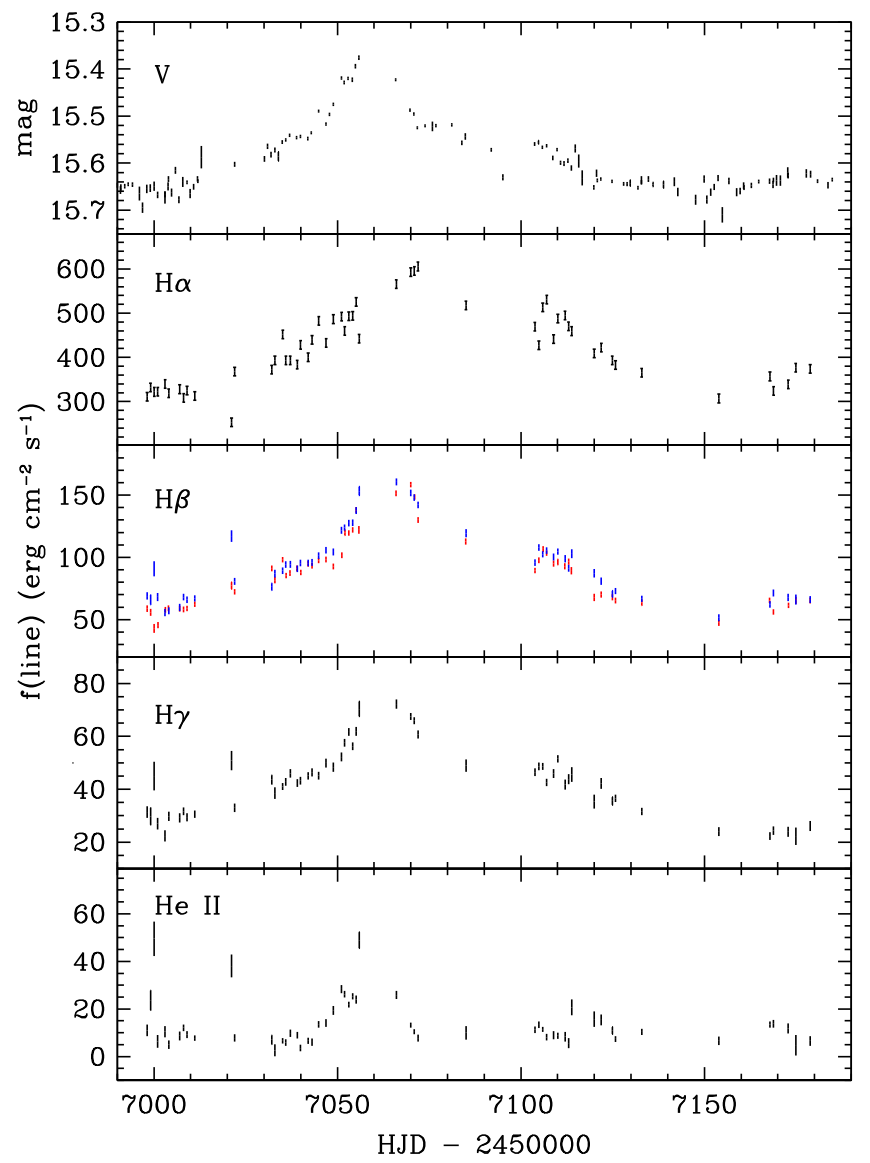

Figure 1. Arp 151 light curves for the $V$-band and the emission lines $\mathrm{H} \alpha, \mathrm{H} \beta$, $\mathrm{H} \gamma$, and $\mathrm{He}$ II as determined from the FLOYDS spectra.The red and blue points show the $\mathrm{H} \beta$ light curve determined from the first- and second-order FLOYDS spectra, respectively, as this emission line is visible in both simultaneously. The normalized excess rms scatter of the [O III] light curve has been added in quadrature to the photon-counting error bars on each data point to take in account of systematic errors.

$3.51 \AA$ pixel $^{-1}$ (resolution $13 \AA$ ), while second order covers $3200-5900 \AA$ at $1.74 \AA$ pixel $^{-1}$ (resolution $7 \AA$ )-for convenience, we will refer to these as the red and blue side, respectively. The significant order overlap allows the $\mathrm{H} \beta$ emission line to be observed in both orders simultaneously, providing a check on our systematic uncertainties related to emission-line measurements. The median continuum signal-tonoise ratio $(\mathrm{S} / \mathrm{N})$ per pixel is 22.5 at $4600 \AA$ on the blue side and 61.2 at $6400 \AA$ on the red side.

The spectroscopic data were batch reduced with a modified version of the FLOYDS data reduction pipeline called AGNFLoYds, written in a PyRAF/Python framework. Briefly, AGNFLOYDS performs standard image detrending (overscan subtraction, flat fielding, and defringing), cosmic-ray rejection (via the L.A. Cosmic algorithm; van Dokkum 2001), order rectification, spectral extraction, and flux and wavelength calibration. Each spectral order is processed separately. We utilize a fixed, unweighted extraction aperture of 8 ". 8 to minimize the effect of varying seeing conditions throughout the campaign. Error spectra were produced and propagated forward for the emission-line light curve measurements. All spectra were reduced without human intervention. 


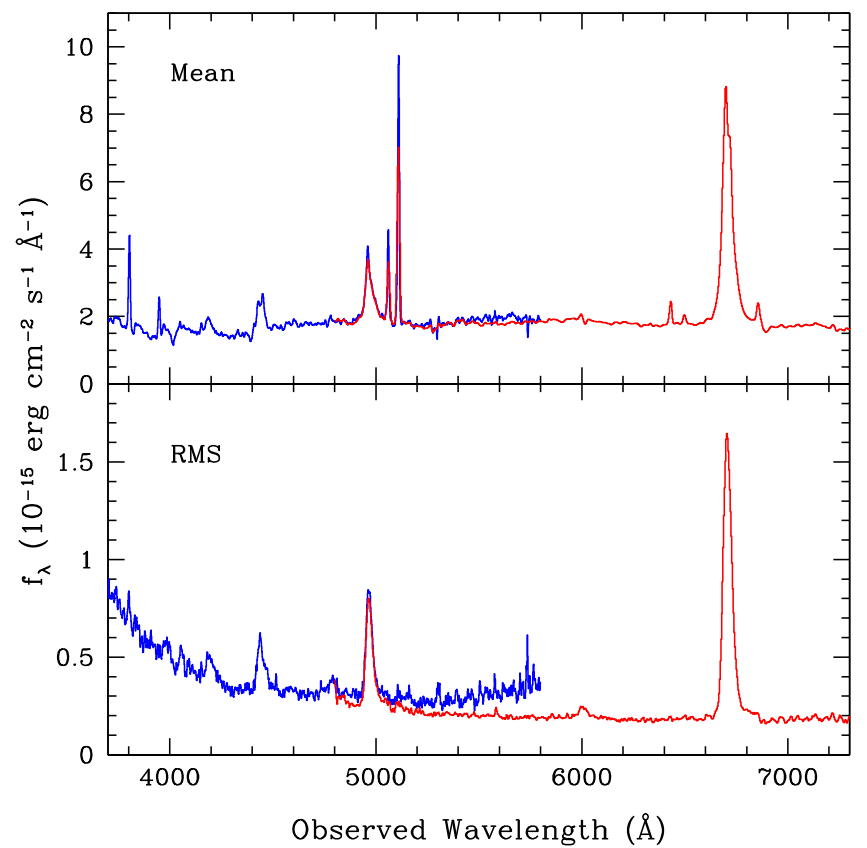

Figure 2. Mean and rms spectra of Arp 151 from FLOYDS, displaying both the blue- and red-side spectra. Note that $\mathrm{H} \beta$ is in the wavelength overlap region of FLOYDS and appears in both first and second order. A small flux scaling was applied to the red-side spectrum to match that of the blue side. The upturn in the red side of the blue rms spectrum is due to the lower $\mathrm{S} / \mathrm{N}$ of the data as the second-order throughput of the grating declines.

\section{EMISSION-LINE LIGHT CURVES}

Emission-line measurements were carried out on the FLOYDS spectra in a similar way as the recent Lick AGN Monitoring Project 2011 reverberation mapping campaign (Barth et al. 2011, 2015), with the blue- and red-side spectra treated separately.

First, the reduced spectra were placed on a uniform flux and wavelength scale utilizing the procedure of van Groningen \& Wanders (1992), which applies a linear wavelength shift, flux scaling factor, and Gaussian broadening term to minimize differences in the spectral region around the $[\mathrm{O}$ III $] \lambda 5007$ line from night to night, under the assumption that this narrow line has a constant flux during the spectroscopic campaign. After this procedure was performed, the excess rms scatter of the [O III] light curve in our spectroscopic time series was $\sim 2 \%$ for both the blue- and red-side spectra, similar to recent reverberation mapping work, which can be considered as a measure of the random error in flux calibration of the final scaled spectra (e.g., Barth et al. 2015). The final mean and rms spectra can be seen in Figure 2, constructed following Peterson et al. (2004); note that the [O III] line almost completely disappears in the rms spectrum of both the red and blue arm, showing the high quality of the data set.

For the blue-side scaled spectra, we performed spectral decomposition over a wavelength range of $\sim 4200-5300 \AA$ in a manner identical to Barth et al. (2015) in order to isolate the $\mathrm{H} \beta, \mathrm{H} \gamma$, and $\mathrm{He}$ II emission lines for our light curve measurements. Briefly, an eight-component model is fit to the daily, scaled spectra utilizing the IDL MPFIT package (Markwardt 2009). The components of the model include: (1) a stellar spectrum with a single-burst age of $11 \mathrm{Gyr}$ and solar metallicity (Bruzual \& Charlot 2003); (2) a power-law AGN featureless continuum component; (3) the [O III] $\lambda 4959,5007$ lines; (4)
Table 1

Cross-correlation Lag Results

\begin{tabular}{lcccc}
\hline \hline Emission Line & $F_{\text {var }}$ & CCF rmax & $\begin{array}{c}\tau_{\text {cen }} \\
\text { (days) }\end{array}$ & $\begin{array}{c}\tau_{\text {peak }} \\
\text { (days) }\end{array}$ \\
\hline $\mathrm{H} \alpha$ & $0.20 \pm 0.02$ & 0.87 & $13.89_{-1.41}^{+1.39}$ & $13.50_{-3.75}^{+4.00}$ \\
$\mathrm{H} \beta$ blue-side & $0.29 \pm 0.03$ & 0.93 & $7.52_{-1.06}^{+1.43}$ & $5.75_{-1.00}^{+0.75}$ \\
$\mathrm{H} \beta$ red-side & $0.32 \pm 0.03$ & 0.93 & $8.59_{-1.21}^{+0.92}$ & $6.00_{-1.25}^{+1.75}$ \\
$\mathrm{H} \gamma$ & $0.30 \pm 0.03$ & 0.91 & $7.40_{-1.32}^{+1.50}$ & $5.75_{-1.25}^{+0.75}$ \\
$\mathrm{He}$ II & $0.70 \pm 0.07$ & 0.47 & $1.83_{-2.58}^{+4.15}$ & $1.00_{-1.75}^{+4.75}$ \\
\hline
\end{tabular}

Note. All lags are measured relative to the $V$-band light curve and are given in the observed frame. All line fluxes include constant narrow-line contributions, slightly decreasing the value of $F_{\text {var }}$.

broad and narrow $\mathrm{H} \beta$; (5) broad and narrow He II; (6) broad $\mathrm{He}$ I; (7) an Fe II blend contribution utilizing the templates of Kovačević et al. (2010); and (8) a Cardelli et al. (1989) reddening law with the color excess $E(B-V)$ as a free parameter. The wavelength region around the $\mathrm{H} \gamma$ and [O III] $\lambda 4363$ lines was masked out from 4280 to $4400 \mathrm{~A}$ during the fitting process due to their complexity (see Barth et al. 2015 for details).

Once the best-fitting spectral decomposition is found, all components except for the broad and narrow $\mathrm{H} \beta$ component were subtracted from each spectrum, leaving only the $\mathrm{H} \beta$ and $\mathrm{H} \gamma$ lines and fitting residuals in the resulting spectra. Light curves of these decomposed spectra were generated via direct integration and can be seen in Figure 1. Note that both of these light curves include a narrow-line component, and the $\mathrm{H} \gamma$ light curve includes a contribution from the [O III] $\lambda 4363$ line. The light curve of the relatively weak $\mathrm{He}$ II $\lambda 4686$ line was determined directly from each night's best-fitting model component spectrum, which includes both broad and narrow components. The Fe II emission proved to be too weak to yield a useful light curve.

For the red-side scaled spectra, we measure both the $\mathrm{H} \beta$ and $\mathrm{H} \alpha$ (which includes contributions from the narrow [ $\left.\mathrm{N}_{\mathrm{II}}\right]$ $\lambda 6548,6583$ lines) fluxes by direct integration after subtracting a simple linear continuum from adjacent line-free regions. The $\mathrm{H} \beta$ light curve derived in this way agrees remarkably well with the FLOYDS blue-side $\mathrm{H} \beta$ light curve, derived via our spectral fits (compare the blue and red points in the $\mathrm{H} \beta$ panel of Figure 1).

The emission-line light curves in Figure 1 show a morphology similar to that seen in the $V$-band light curve, with a factor of 3 rise and decline during the $\sim 200$ day period of our campaign. For all of our emission-line light curves, we have measured the normalized rms variability amplitude $F_{\text {var }}$ (e.g., Peterson et al. 2004). We present these values in Table 1 . In general, values of $F_{\mathrm{var}}>0.1$ correspond to strong line variations that are amenable to accurate lag measurement (provided that the light curve exhibits sufficient structure rather than a simple monotonic increase or decrease in flux). The light curves for $\mathrm{H} \alpha, \mathrm{H} \beta, \mathrm{H} \gamma$, and $\mathrm{He}$ II all exhibit variations well in excess of this threshold.

We also note that as a consistency check we derived emission-line light curves using the software suite PREPSPEC, developed by K. Horne (see, e.g., Grier et al. 2013 for a description of the software), and found similar light curves for all the lines measured here. 


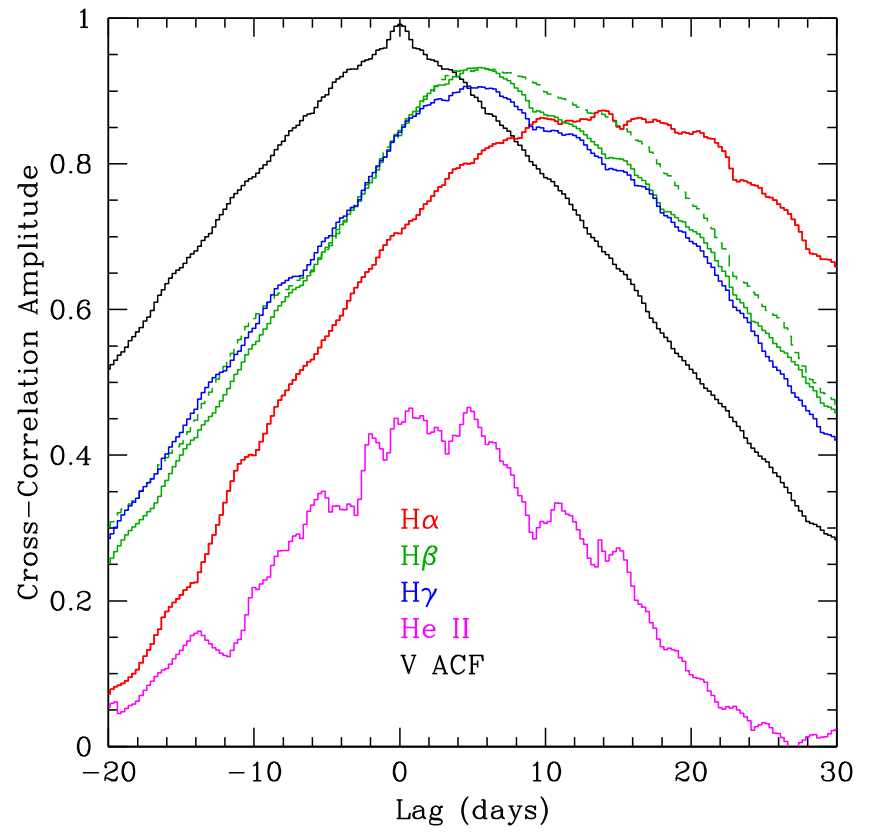

Figure 3. Cross-correlation functions of $\mathrm{H} \alpha, \mathrm{H} \beta$ (solid line is the blue-side data, dashed line is from the red side), $\mathrm{H} \gamma$, and $\mathrm{He}$ II against the $V$-band light curve. Also shown is the autocorrelation function of the $V$-band continuum.

\section{REVERBERATION LAG MEASUREMENTS}

We employ standard cross-correlation function (CCF) techniques, utilizing the interpolation and Monte Carlo error analysis methodology of previous reverberation mapping workers (e.g., White \& Peterson 1994; Peterson et al. 2004; Bentz et al. 2009; Denney et al. 2010), in order to measure robust lags in our data set. We measured cross-correlations of $\mathrm{H} \alpha, \mathrm{H} \beta$ (on the red- and blue-side spectra independently), $\mathrm{H} \gamma$, and $\mathrm{He}$ II against the $V$-band light curve from -20 to +40 days in increments of 0.25 days. We show the CCF measurements in Figure 3 and present the results in Table 1 . In Table 1, $\tau_{\text {peak }}$ corresponds to the peak of the $\mathrm{CCF}$ and $\tau_{\mathrm{cen}}$ corresponds to the centroid of the CCF for all points above $80 \%$ of the peak value (Peterson et al. 2004).

First, the blue- and red-side $\mathrm{H} \beta$ lags are in excellent agreement, with $\tau_{\text {cen }}=7.52_{-1.06}^{+1.43}$ and $8.59_{-1.21}^{+0.92}$ days, respectively, lending confidence to our data reduction and analysis techniques. The $\mathrm{H} \gamma$ lag of $\tau_{\text {cen }}=7.40_{-1.32}^{+1.50}$ days is consistent with the $\mathrm{H} \beta$ measurements, while the He II lag is consistent with zero $\left(\tau_{\text {cen }}=1.83_{-2.58}^{+4.15}\right.$ days). We also measure an $\mathrm{H} \alpha$ lag, $\tau_{\mathrm{cen}}=13.89_{-1.41}^{+1.39}$ days, that is substantially larger than that of $\mathrm{H} \beta$. The relative lags between each line are consistent with that seen in other reverberation mapped systems, along with previous measurements of Arp 151 (e.g., Bentz et al. 2010b). While the 2015 LCOGT reverberation lags are a factor of $\sim 2$ larger than the lags previously measured by Bentz et al. (2010b) from the 2008 Lick campaign, Arp 151's $\mathrm{H} \beta$ velocity width is smaller than measured in the 2008 data, leading to virtually the same virial products-see Section 6.

Past data on Arp 151 have demonstrated velocity-resolved variability and lags (Bentz et al. 2008, 2010a; Pancoast et al. 2014b). We measured $\mathrm{H} \beta$ light curves in seven velocity bins across the width of the line (utilizing our blue-side FLOYDS data) and cross-correlated each with the $V$-band light curve. We plot our velocity-resolved lag results $\left(\tau_{\text {cen }}\right)$ in Figure 4 , which shows a similar morphology as previous velocity-resolved

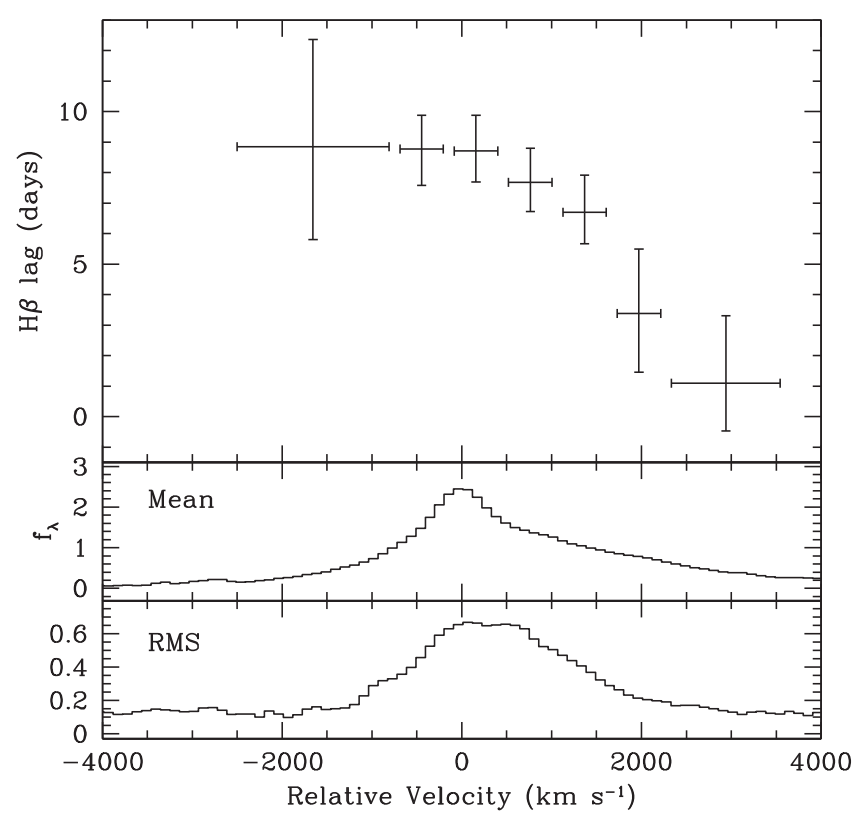

Figure 4. Velocity-resolved $\mathrm{H} \beta$ lags, as derived from our blue-side FLOYDS data. The upper panel shows the lag (using the $\tau_{\text {cen }}$ measure) for each velocity bin, where the horizontal error bar represents the bin size. The bottom panels show the mean and rms continuum-subtracted spectra.

work in Arp 151. The $\mathrm{H} \beta$ lag response is clearly asymmetric, with an $\sim 8$ day difference between the lag in the $\mathrm{H} \beta$ core and the red wing of the line. The lag profile is similar in shape to that seen in the 2008 Lick data (Bentz et al. 2008, 2010a), despite the factor of $\sim 2$ increase in the overall $\mathrm{H} \beta$ lag since 2008. A symmetric $\mathrm{H} \beta$ lag response, declining on both the blue and red wings of the line, would be consistent with the BLR clouds in Keplerian, circular orbits around the black hole (Welsh \& Horne 1991). However, the velocity lags such as that seen in Arp 151, with higher lags on the blue wing, with a decline toward the red, are generally consistent with BLR models with radial inflow and/or a warped disk morphology (e.g., Bentz et al. 2010a; Pancoast et al. 2014a).

\section{LINE WIDTHS AND BLACK HOLE MASS ESTIMATE}

The width of the broad $\mathrm{H} \beta$ line is needed for determining black hole masses from reverberation data, which we measure following the procedure of Barth et al. (2015). First, we use the results of the spectral decompositions of the nightly secondorder FLOYDS spectra to remove all components except for the broad $\mathrm{H} \beta$ emission from the data. From these broad $\mathrm{H} \beta$ only spectra, we construct mean and rms spectra and measure the line width parameters following the methods of Peterson et al. (2004). Uncertainties are calculated via a Monte Carlo resampling technique. The instrumental resolution of FLOYDS with the 1".6 slit near the $\mathrm{H} \beta$ line is $\mathrm{FWHM}=7 \AA$, corresponding to $\approx 430 \mathrm{~km} \mathrm{~s}^{-1}$ (or $\sigma_{\text {inst }} \approx 183 \mathrm{~km} \mathrm{~s}^{-1}$ ), and we subtract this small contribution in quadrature for our final results. The broad $\mathrm{H} \beta$ from the mean spectrum has a FWHM $\left(\mathrm{H} \beta_{\text {mean }}\right)=2872 \pm 90 \mathrm{~km} \mathrm{~s}^{-1}\left[\sigma_{\text {line }}\left(\mathrm{H} \beta_{\text {mean }}\right)=1591 \pm 55 \mathrm{~km}\right.$ $\mathrm{s}^{-1}$, while for the rms spectrum it is $\operatorname{FWHM}\left(\mathrm{H} \beta_{\mathrm{rms}}\right)=1833 \pm$ $61 \mathrm{~km} \mathrm{~s}^{-1}\left[\sigma_{\text {line }}\left(\mathrm{H} \beta_{\mathrm{rms}}\right)=879 \pm 57 \mathrm{~km} \mathrm{~s}^{-1}\right]$.

The measured $\mathrm{H} \beta$ virial product, defined as $\mathrm{VP}=R_{\mathrm{BLR}}(\Delta V)^{2} / G$, is determined by using $\Delta V=\sigma_{\text {line }}\left(\mathrm{H} \beta_{\mathrm{rms}}\right)$ and $R_{\mathrm{BLR}}=c \tau_{\text {cen }}$ (see Peterson et al. 2004), which we find to be $\mathrm{VP}=1.13_{-0.22}^{+0.26} \times 10^{6} M_{\odot}$. This value for the virial product is 
consistent to within the uncertainties with previous determinations of $M_{\mathrm{BH}}$ in Arp 151 (e.g., Bentz et al. 2010b), which also indicates that $\sigma_{\text {line }} \propto \tau^{-0.5}$, as is expected for a virial relationship between time lag and line width.

To convert our directly measured virial product into a black hole mass requires an additional dimensionless factor, $f$, which depends on the geometry, kinematics, and orientation of the BLR. While recent work has made great progress in directly determining the value of $f$ for individual AGNs (Brewer et al. 2011; Pancoast et al. 2011, 2012, 2014a, 2014b), we adopt a value of $f=5.5$, which was found by Onken et al. (2004) to bring the AGN $M_{\mathrm{BH}}-\sigma_{*}$ relationship into agreement with the same relationship for quiescent galaxies. We then find $M_{\mathrm{BH}}=6.2_{-1.2}^{+1.4} \times 10^{6} M_{\odot}$, where the quoted uncertainty comes only from the uncertainty on the virial product and not on the adopted value of $f$. As $M_{\mathrm{BH}}$ is simply the virial product multiplied by the $f$-factor, this result is also in agreement with previous work on Arp 151 and consistent with Brewer et al. (2011) and Pancoast et al. (2014b) within the uncertainties.

\section{SUMMARY AND FUTURE PROSPECTS}

We have presented the first totally robotic AGN reverberation mapping measurements for the nearby Seyfert 1 galaxy Arp 151 as part of the LCOGT AGN Key Project. Robust lag measurements were measured for the $\mathrm{H} \beta, \mathrm{H} \alpha$, and $\mathrm{H} \gamma$ lines, and we were able to measure a velocity-resolved $\mathrm{H} \beta$ lag with a profile similar to that seen in previous work. By combining the lag measurements with line width measurements, we infer a central black hole mass of $M_{\mathrm{BH}}=6.2_{-1.2}^{+1.4} \times 10^{6} M_{\odot}$, again in accordance with previous measurements in this system. Arp 151 is now one of just a few AGNs where it has been shown that the virial product remains constant against substantial changes in $\mathrm{H} \beta$ lag and luminosity. NGC 5548 is the best example with many years of monitoring and an order of magnitude variation in luminosity over the years (Bentz et al. 2007).

The reverberation mapping portion of the LCOGT AGN Key Project has collected data on other low-redshift AGNs as well as higher-redshift quasars. In particular, we are monitoring a sample of moderate- and high-redshift objects with a focus on the $\mathrm{Mg}_{\mathrm{II}}$ and $\mathrm{C}_{\mathrm{IV}}$ emission lines and their lags in order to determine a direct $R_{\mathrm{BLR}}-L$ relation for these emission lines. The robotic nature of the LCOGT network and the large allocation of telescope resources are both critical for the success of the project.

Research by A.J.B. and L.P. are supported by NSF grant AST-1412693. Research by D.J.S. is supported by NSF grant AST-1412504 and AST-1517649. Research by T.T. is supported by NSF grant AST-1412315 and a Packard Research Fellowship. E.R.C. and S.C. gratefully acknowledge the receipt of research grants from the National Research Foundation (NRF) of South Africa.

\section{REFERENCES}

Barth, A. J., Bennert, V. N., Canalizo, G., et al. 2015, ApJS, 217, 26 Barth, A. J., Nguyen, M. L., Malkan, M. A., et al. 2011, ApJ, 732, 121 Bentz, M. C., Denney, K. D., Cackett, E. M., et al. 2007, ApJ, 662, 205 Bentz, M. C., Denney, K. D., Grier, C. J., et al. 2013, ApJ, 767, 149 Bentz, M. C., Horne, K., Barth, A. J., et al. 2010a, ApJL, 720, L46 Bentz, M. C., Walsh, J. L., Barth, A. J., et al. 2008, ApJL, 689, L21 Bentz, M. C., Walsh, J. L., Barth, A. J., et al. 2009, ApJ, 705, 199 Bentz, M. C., Walsh, J. L., Barth, A. J., et al. 2010b, ApJ, 716, 993 Blandford, R. D., \& McKee, C. F. 1982, ApJ, 255, 419 Brewer, B. J., Treu, T., Pancoast, A., et al. 2011, ApJL, 733, L33 Brown, T. M., Baliber, N., Bianco, F., et al. 2013, PASP, 125, 1031 Bruzual, G., \& Charlot, S. 2003, MNRAS, 344, 1000

Cardelli, J. A., Clayton, G. C., \& Mathis, J. S. 1989, ApJ, 345, 245 Denney, K. D., Peterson, B. M., Pogge, R. W., et al. 2010, ApJ, 721, 715 Du, P., Hu, C., Lu, K.-X., et al. 2014, ApJ, 782, 45

Grier, C. J., Peterson, B. M., Horne, K., et al. 2013, ApJ, 764, 47

Grier, C. J., Peterson, B. M., Pogge, R. W., et al. 2012, ApJ, 755, 60

Henden, A. A., Levine, S. E., Terrell, D., Smith, T. C., \& Welch, D. 2012, JAVSO, 40, 430

Kovačević, J., Popović, L. Č., \& Dimitrijević, M. S. 2010, ApJS, 189, 15

Lang, D., Hogg, D. W., Mierle, K., Blanton, M., \& Roweis, S. 2010, AJ, 139,1782

Markwardt, C. B. 2009, in ASP Conf. Ser., 411, Astronomical Data Analysis Software and Systems XVIII, ed. D. A. Bohlender, D. Durand, \& P. Dowler (San Francisco, CA: ASP), 251

Onken, C. A., Ferrarese, L., Merritt, D., et al. 2004, ApJ, 615, 645

Pancoast, A., Brewer, B. J., \& Treu, T. 2011, ApJ, 730, 139

Pancoast, A., Brewer, B. J., \& Treu, T. 2014a, MNRAS, 445, 3055

Pancoast, A., Brewer, B. J., Treu, T., et al. 2012, ApJ, 754, 49

Pancoast, A., Brewer, B. J., Treu, T., et al. 2014b, MNRAS, 445, 3073

Pei, L., Barth, A. J., Aldering, G. S., et al. 2014, ApJ, 795, 38

Peterson, B. M. 1993, PASP, 105, 247

Peterson, B. M., Ferrarese, L., Gilbert, K. M., et al. 2004, ApJ, 613, 682

Shen, Y. 2013, BASI, 41, 61

van Dokkum, P. G. 2001, PASP, 113, 1420

van Groningen, E., \& Wanders, I. 1992, PASP, 104, 700

Welsh, W. F., \& Horne, K. 1991, ApJ, 379, 586

White, R. J., \& Peterson, B. M. 1994, PASP, 106, 879 